\title{
CONOCIMIENTO TECNOLÓGICO, PEDAGÓGICO Y DISCIPLINARIO DEL PROFESORADO: EL CASO DE UN CENTRO EDUCATIVO INTELIGENTE
}

\author{
TECHNOLOGICAL, PEDAGOGICAL AND DISCIPLINARY KNOWLEDGE OWNED BY \\ TEACHERS: THE CASE OF AN INTELLIGENT EDUCATIONAL CENTER
}

\author{
Rosabel Roig Vila, rosabel.roig@ua.es \\ Universidad de Alicante (España) \\ Carolina Flores Lueg, cflores@ubiobio.cl \\ Universidad del Bío-Bío (Chile)
}

\section{RESUMEN}

En este estudio se analiza la integración de las TIC en un escenario de aprendizaje específico: un centro público de Alicante catalogado como Centro Educativo Inteligente [CEI], según el programa de pilotaje homónimo de la Comunidad Valenciana. Se pondera detenidamente, mediante una investigación mixta el conocimiento tecnológico, pedagógico y disciplinar de los docentes a partir del Modelo TPACK. Como instrumentos de evaluación se han utilizado encuestas, entrevistas y grupos de discusión. Entre los resultados alcanzados, destaca la constatación de la inseguridad del profesorado al aplicar los conocimientos tecnológico-pedagógicos en sus clases, si bien se trata de un CEI. Se analiza detenidamente la paradoja establecida entre tal percepción del profesorado y la opinión del equipo directivo, que postula lo contrario.

Palabras clave: TIC, Modelo TPACK, Centro Educativo Inteligente.

\begin{abstract}
The present paper has as its main purpose to analyze ICT integration within a specific learning environment: a public center located in Alicante and catalogued as an 'Intelligent Educational Center' (CEI, for its initials in Spanish), according to the homonymous piloting plan designed by the Valencian Autonomous Region (Spain). A mixed research work based on the TPACK model was used to perform a thorough study of technological, pedagogical and disciplinary knowledge. Surveys based on questionnaires and interviews along with discussion groups were our assessment tools. Among the results obtained stands out the verification of the teaching staff's insecurity when it comes to the practical application of technological and pedagogical knowledge in their classes -even though the research was carried out in a CEI. The paradox created by the sharp contrast between that widespread perception among the teaching staff and the completely different view which prevails among school management teams has equally received special attention in our paper.
\end{abstract}

Keywords: ICT in Education, TPACK Model, Intelligent Educational Center. 


\section{INTRODUCCIÓN}

Las Tecnologías de la Información y la Comunicación (TIC) se consideran como recursos que pueden contribuir significativamente a innovar y plantear nuevos procesos culturales y educacionales. Desde esta perspectiva, las políticas educativas se han dirigido hacia la implementación de diversos programas orientados a incorporar las TIC en todos los niveles del sistema educativo con el fin de alfabetizar digitalmente a niños y jóvenes, lo que ha dado lugar a la ejecución de grandes inversiones en equipamiento tecnológico e infraestructuras y en programas de formación del profesorado, entre otras acciones, tales como la creación de directorios de materiales digitales.

Uno de los programas impulsados por el Ministerio de Educación español fue el proyecto Escuela 2.0 en el curso 2009-10, una iniciativa que pretendía ofrecer una propuesta común para la integración de las TIC en los centros educativos públicos y concertados de todas las Comunidades Autónomas. Al respecto, es importante destacar que la Comunidad de Madrid y la Comunidad Valenciana decidieron no sumarse a esta iniciativa, si bien esta última, a partir del mismo año, comienza a implementar una propuesta paralela denominada Centros Educativos Inteligentes (CEI). Ésta consistía en dotar de tecnología las aulas de los centros tanto las ordinarias y de informática como las específicas -, aulas móviles, conectividad a Internet y formación del profesorado. En 2010, pues, se establece la organización y funcionamiento del programa, para lo cual se seleccionaron 18 centros educativos públicos de la Comunidad que se constituirían en centros piloto (DOGV, 2010) y cuya experimentación delimitaría el escenario de aprendizaje modelo para el resto de centros.

A pesar de estas y otras acciones emprendidas, cabe decir que el proceso de integración de las TIC en los centros educativos ha sido más lento que en otras instituciones sociales, pues existen ciertas dificultades que se deben tener en cuenta. Al respecto, Donohoo (2004) (cit. en Silva \& Astudillo, 2012, p. 3) señala que las barreras manifestadas por las escuelas para integrar las TIC se pueden agrupar en tres categorías: el profesor, la infraestructura y lo social, donde el papel del profesor sería el factor más importante para el éxito de la integración de estas herramientas. Por su parte, Valverde, Garrido y Sánchez (2010, p. 208), señalan que, por lo general, el profesorado interpreta las TIC con sus propios prejuicios y predilecciones, vinculados a las formas de uso y aplicación que consideran "apropiadas" e "inapropiadas". Por lo tanto, el significado y sentido que le atribuye el profesorado a las TIC responde a la forma en cómo va construyendo su acercamiento cognitivo, afectivo y conductual frente a los objetos tecnológicos, específicamente en lo referido a su uso e incorporación dentro de sus prácticas educativas.

Asimismo, algunos autores (Domínguez, 2011; Gallardo, Suárez \& Almerich, 2006; López, 2011; Marín \& Reche, 2012; Ramírez, Cañedo \& Clemente, 2012; Silva \& Astudillo, 2012; Tejedor \& García-Valcárcel, 2006) han abordado la actitud del profesorado desde diversas perspectivas. Los resultados, en general, dan cuenta de las actitudes positivas que presenta el profesorado para con las TIC, aunque están vinculadas a la percepción que tienen sobre sus niveles de competencia digital y de conocimientos tecnológicos.

Consideramos, pues, la formación del profesorado como factor decisivo en la integración de las TIC en los centros educativos. Tiene, por tanto, su razón de ser que, en los programas 
específicos con esta finalidad, se incorpore dicha formación. Del mismo modo, se entenderá que la implementación de estos programas repercuta en la formación óptima en TIC del profesorado. En lo que respecta a dicha formación, un propuesta teórica que orienta sobre los aspectos centrales a tener en cuenta en la incorporación de estas herramientas en el proceso formativo es el Modelo TPACK o "Technological Pedagogical Content Knowledge" (Harris, Mishra \& Koehler, 2009; Mishra \& Koehler, 2006; Sing, Hwee, \& Tsai, 2013). Este modelo identifica tres dimensiones del conocimiento básico (véase fig. 1): Conocimiento Disciplinar (CK, Content Knowledge), Conocimiento Pedagógico (PK, Pedagogical Knowledge) y Conocimiento Tecnológico (TK, Technological Knowledge), pero el énfasis está puesto en las nuevas formas de conocimiento que se generan, a su vez, en la intersección entre cada uno de estos conocimientos -TCK, PCK y TPK-, para llegar a la intersección de las tres dimensiones que determina la situación óptima -TPACK-.

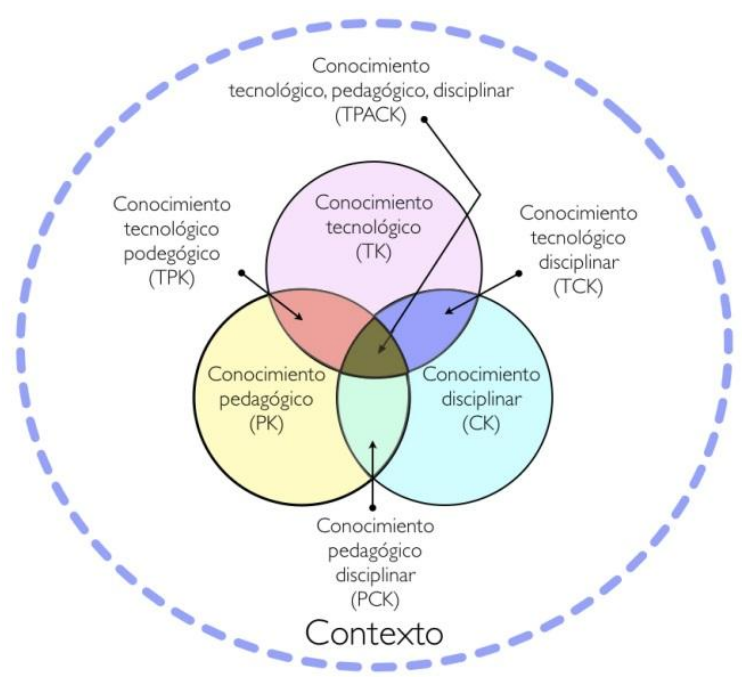

Fig 1. Esquema del modelo TPACK.

En este marco contextual, nos propusimos realizar una investigación en uno de los centros pilotos del programa Centros Educativos Inteligentes de la Generalitat Valenciana. Se trataba de analizar, teniendo en cuenta el modelo TPACK, cuál era la autovaloración del profesorado con respecto a su formación después de haber implementado en su centro el programa referido. Así, nos planteamos como objetivos conocer el conocimiento tecnológico, pedagógico y disciplinario de los docentes sobre la integración de las TIC en la enseñanza e indagar acerca de las opiniones y experiencias educativas del profesorado sobre el uso de las TIC en el aula. De forma complementaria, hemos analizado la opinión del equipo directivo respecto a estas cuestiones, con el fin de delimitar con más precisión el objeto de estudio.

\section{METOdOLOGÍA}

A partir de los objetivos planteados, se consideró como método de investigación un estudio de casos, entendido éste como un proceso de indagación caracterizado por el examen sistemático y en profundidad de entidades sociales o entidades educativas únicas (Bisquerra, 2009), por lo tanto, los resultados de este estudio sólo son posibles de ser interpretados a partir de la valoración y los significados que los actores consultados construyen en torno a las TIC aplicadas al proceso educativo en la realidad estudiada. Para abordar el objeto de 
estudio se consideró la complementariedad metodológica, pues se concuerda con Sáez y Touriñán (2012, p. 113) cuando señalan que "las investigaciones más completas serán aquellas que se ocupan de la multidimensionalidad de los objetos de estudio y los abordan tanto cualitativa como cuantitativamente".

El contexto de estudio correspondió a un centro educativo de educación infantil y primaria (C.E.I.P) ubicado en la localidad de El Campello (Alicante) seleccionado en el programa de pilotaje de Centros Educativos Inteligentes [CEI] de la Comunidad Valenciana. Desde la dirección del centro se realizaron las gestiones para poder desarrollar el estudio, el cual se llevó a cabo durante el curso 2012-13: se informó al profesorado de los objetivos propuestos y se solicitó la participación de forma voluntaria. La muestra, pues, fue seleccionada de forma incidental o de conveniencia, y la conformaron 29 profesores -de 41 que tiene el centro- Participaron el $71 \%$ de los docentes del centro, donde el $28 \%$ corresponde a hombres y un $72 \%$ a mujeres. La media de edad es de 37,7 años y la de años de experiencia docente es de 13,4 años (ver Tabla 1)

\begin{tabular}{|l|r|r|r|r|c|}
\hline Variables & N & Mínimo & Máximo & Media & $\begin{array}{c}\text { Desv. } \\
\text { típ. }\end{array}$ \\
\hline Años de experiencia docente & 26 & 0 & 30 & 13,44 & 7.91 \\
Edad & 26 & 24 & 55 & 37,69 & 8,84 \\
N válido (según lista) & 3 & & & & \\
\hline
\end{tabular}

Tabla 1: Información sociodemográfica del profesorado.

El cuestionario empleado para evaluar la autovaloración de los conocimientos adquiridos respecto a la integración de las TIC fue una versión traducida y simplificada del cuestionario original de Schmidt, Baran, Thompson, Mishra, Koehler y Shin (2009) para analizar los conocimientos de los docentes según el modelo TPACK. El cuestionario que se administró en esta investigación abarca las mismas dimensiones que el cuestionario original: Conocimiento Tecnológico (KT) - Conocimiento Pedagógico (PK)- Conocimiento Disciplinar (CK) Conocimiento Tecnológico-Pedagógico (TPK) - Conocimiento Tecnológico-Disciplinar (TCK) y Conocimiento Tecnológico-Pedagógico aplicado al Contenido (TPACK). Los ítems de respuesta estaban en formato Likert, con valoraciones del 1 (totalmente en desacuerdo) al 5 (totalmente de acuerdo). Contiene 29 indicadores centrados en la tipología de conocimientos TPACK: TK, ítems 1, 2, 3, 4, 5, 6, 7; CK, ítems 8, 9, 10; PK, ítems 11, 12, 13, 14, 15, 16, 17; PCK, ítem 18; TCK, ítem 19; TPK, ítems 20, 21, 22, 23, 24; TPACK, ítems 25, 26, 27, 28, 29.

Se realizó un focus group con la participación de cinco profesores de los diversos niveles educativos del centro. Las categorías consideradas fueron: Percepción sobre el uso de las TIC - Tecnología y metodología - Aspectos a mejorar. Este focus group tuvo una duración aproximada de una hora, fue grabado en archivo de audio y transcrito posteriormente a un archivo de texto respetando el discurso emitido por los participantes.

De forma paralela se llevó a cabo una entrevista semiestructurada con cada miembro del equipo directivo del centro: Director y Jefa de Estudios, con la finalidad de detectar aspectos referidos al escenario organizativo para el aprendizaje y delimitar las características del 
Centro Inteligente. Las entrevistas tuvieron una duración aproximada de $\mathbf{3 0}$ minutos, fueron transcritas respetando plenamente el discurso de estos actores y posteriormente analizadas sobre la base de las siguientes categorías semánticas: Percepción sobre el uso de las TICAspectos a mejorar - Características del centro Educativo y Políticas de incorporación de las TIC.

Los documentos generados a partir del focus group y las entrevistas fueron analizados con el sofware AtlasTi. v. 7. El procedimiento seguido contempló las siguientes fases:

- Delimitación de las categorías según un modelo emergente y a partir de la lectura de las transcripciones se llevó a cabo la codificación de cada texto.

- Organización de las categorías en una tabla de co-ocurrencia de códigos.

- Generación de redes de nodos.

- Análisis y extracción de conclusiones a partir de los fragmentos codificados.

Los análisis de los cuestionarios aplicados a los docentes se llevaron a cabo por medio de estadísticos descriptivos. Para velar por la calidad de la investigación nos planteamos la triangulación entre instrumentos y la triangulación metodológica.

\section{RESULTADOS E INTERPRETACION}

\subsection{Autovaloración que posee el profesorado sobre sus conocimientos tecnológicos,} pedagógicos y disciplinares relacionados con la integración de las TIC.

A partir de los resultados obtenidos en el cuestionario aplicado a los profesores cabe decir, de forma global, que en su mayoría los profesores manifiestan tener conocimiento sobre los contenidos que imparten y saber aplicarlos, pero se aprecia un comportamiento distinto en los ítems referidos al conocimiento tecnológico, pues el porcentaje que contesta de forma positiva disminuye considerablemente, principalmente en lo que respecta al conocimiento sobre diferentes elementos tecnológicos, matenerse al día en cuanto a las nuevas tecnologías, aprender de forma rápida en estos temas y saber resolver problemas técnicos (véase gráf. 1).

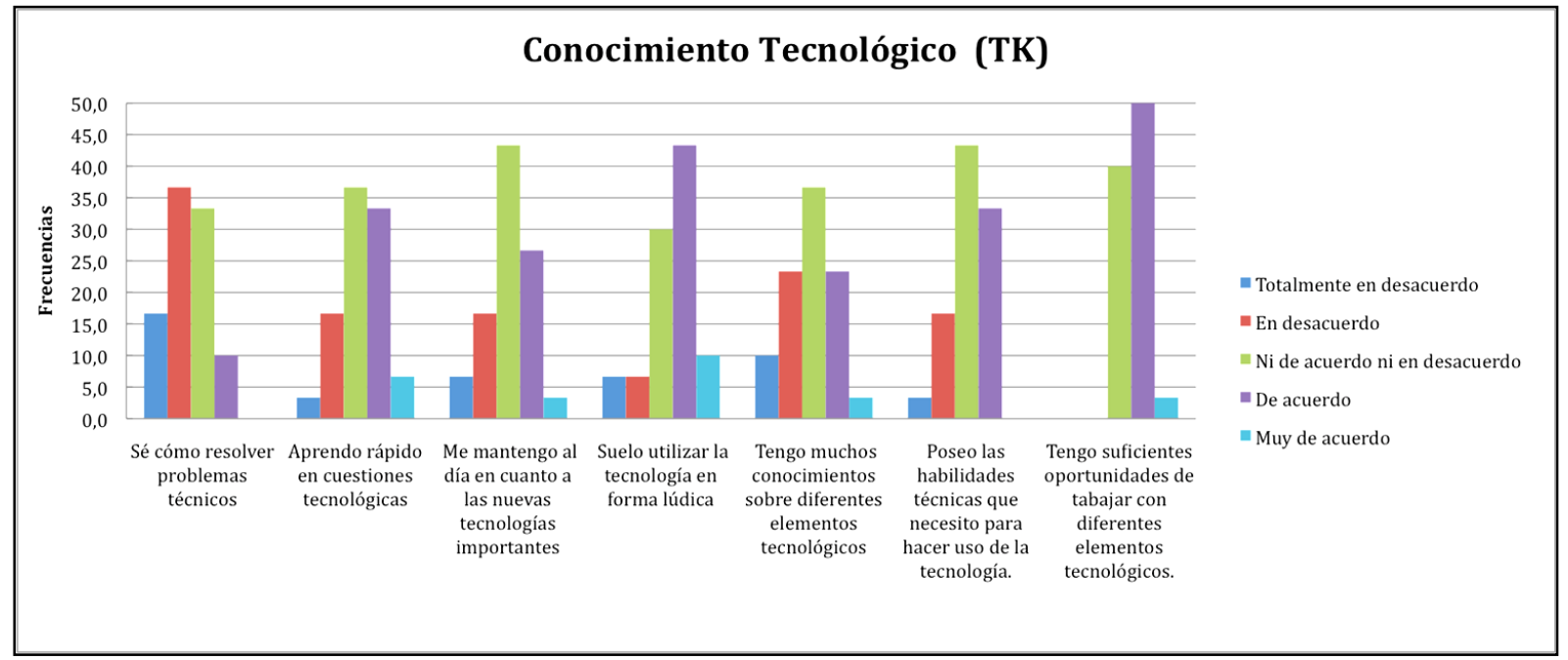

Gráfico 1. Conocimiento Tecnológico del profesorado 
Con respecto al conocimiento disciplinario (véase gráf. 2), se observa que los profesores consultados sienten, de forma mayoritaria, que tienen suficientes conocimientos sobre la materia que imparten, aunque llama la atención que un $26,7 \%$ no autoperciba claramente este aspecto. Los dos ítems restantes han dado como resultado porcentajes muy altos en cuanto al nivel de afirmación -que contrasta claramente con los resultados del gráfico 1 referido al conocimiento tecnológico.

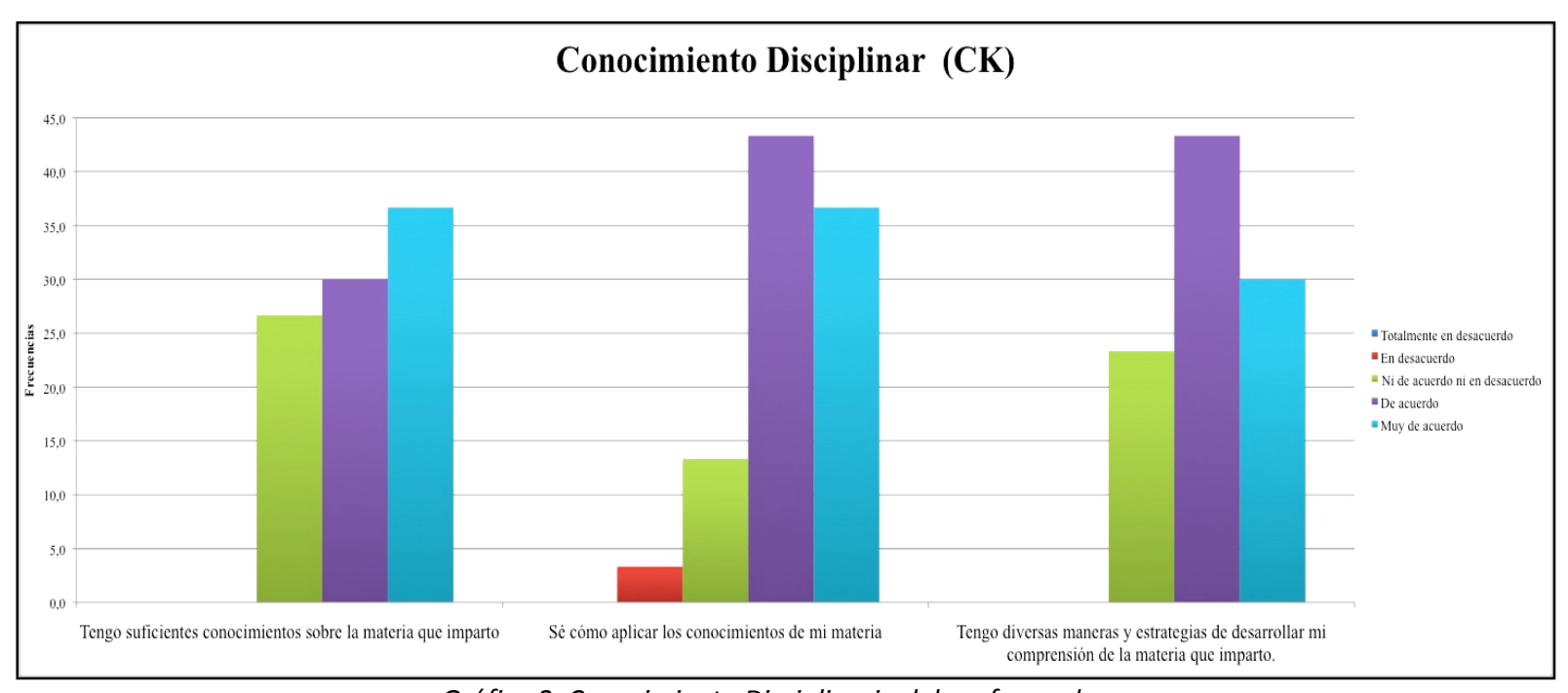

Gráfico 2. Conocimiento Disciplinario del profesorado.

Se aprecia una valoración positiva por parte del profesorado en lo que respecta al conocimiento pedagógico (véase gráf. 3), pues las frecuencias más altas se agrupan en las categorías "totalmente de acuerdo" y "de acuerdo". No obstante, llama la atención las respuestas intermedias de algunos docentes en torno a cuestiones sobre enfoques didácticos o estilos de aprendizaje, aspectos que corresponden a saberes básicos del desempeño profesional del docente.

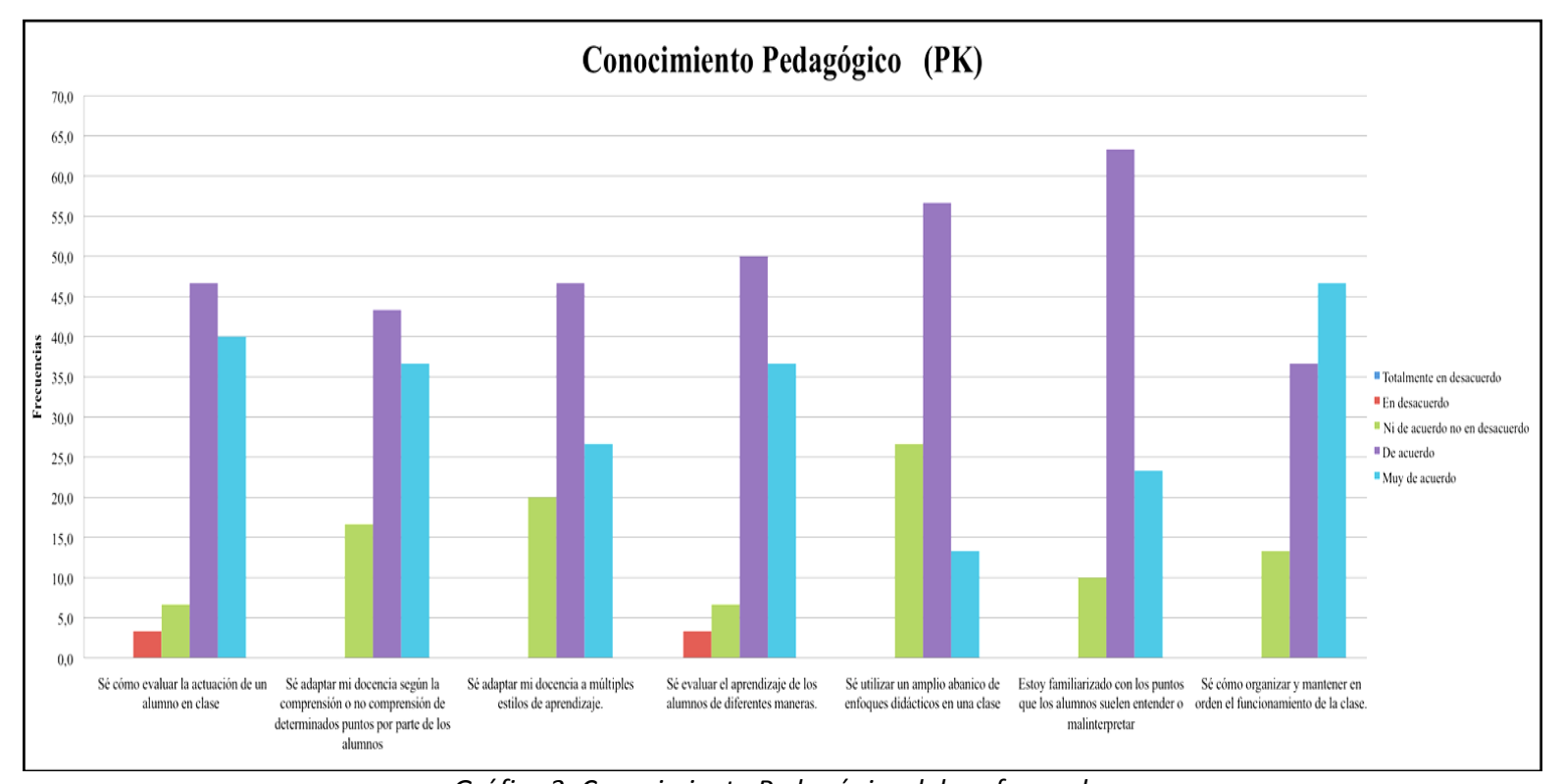

Gráfico 3: Conocimiento Pedagógico del profesorado 
Con respecto al único ítem referido específicamente al conocimiento pedagógico disciplinar (PCK) (Sé elegir enfoques didácticos efectivos para guiar el razonamiento y el aprendizaje de los alumnos en la materia que imparto), un $23,3 \%$ contesta que está totalmente de acuerdo con esta afirmación y un $46,7 \%$ de acuerdo. Son valores, pues, altos los referidos al conocimiento que todo maestro utiliza al enseñar un contenido determinado.

Del mismo modo, en cuanto a la dimensión conocimiento tecnológico disciplinar (TCK) hay un único ítem en el cuestionario. El profesorado manifiesta estar de acuerdo (50\%) y muy de acuerdo (10\%) con la afirmación: Conozco elementos tecnológicos que puedo utilizar para mejorar la comprensión y aplicar en clase. Un 33,3\% presenta una postura imparcial y un 3,3\% no está de acuerdo. Estos dos últimos resultados llaman la atención dadas las características que presenta el centro educativo donde ejercen su labor, pues al ser un Centro Inteligente la infraestructura en cuanto a recursos tecnológicos es muy significativa.

Respecto al conocimiento tecnológico-pedagógico (TPK) que pone el énfasis en el conocimiento de las características y el potencial de las múltiples tecnologías utilizadas en contextos de enseñanza aprendizaje, se puede apreciar (véase gráf.4) que en los ítems referidos a considerar críticamente cómo utilizar la tecnología en clase y el poder adaptar los elementos tecnológicos a diferentes actividades didácticas, el $63,4 \%$ y el $63,3 \%$ respectivamente del profesorado manifiesta estar muy de acuerdo o de acuerdo con ello, siendo estos los ítems mejor valorados. Si bien es cierto en los otros ítems medidos también se observan porcentajes mayores que el $50 \%$ en ambas categorías, los porcentajes de profesores que señalan sentirse indiferentes o que están en desacuerdo aumenta significativamente. También hacemos notar los resultados negativos obtenidos en el ítem referido a la formación inicial con respecto a este tipo de conocimiento (Mi carrera me ha ayudado a pensar más profundamente en cómo la tecnología podría influir en los enfoques didácticos que utilizo en clase).

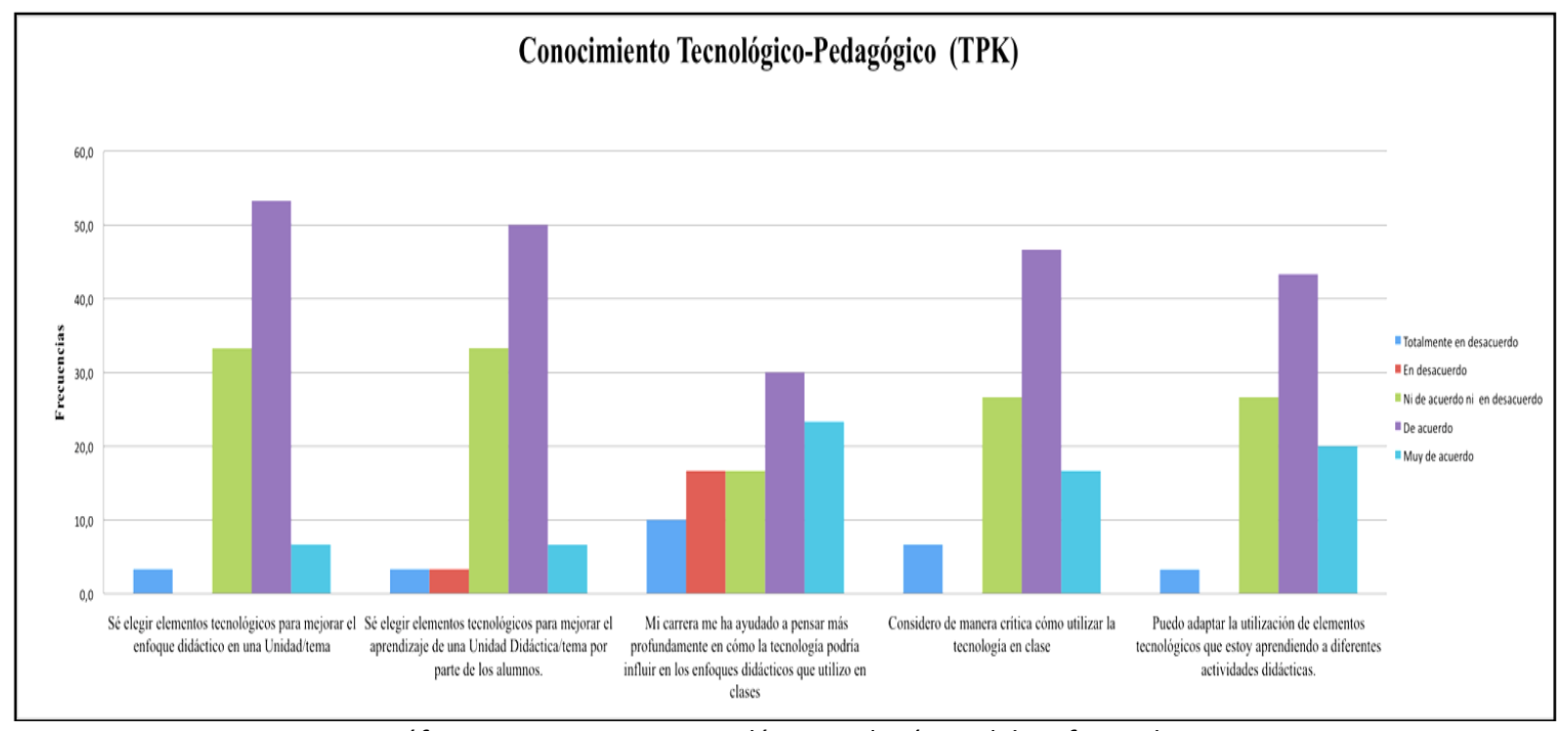

Gráfico 4: Conocimiento Tecnológico-Pedagógico del profesorado

Analizando los resultados sobre la intersección entre los tres tipos de conocimiento, es decir, el conocimiento tecnológico-pedagógico aplicado a los contenidos (TPACK) (véase gráf. 5), se observa que el porcentaje de profesores que responde positivamente en cada uno de los 
ítems es bastante bajo, pues, sólo el $53 \%$ de ellos está de acuerdo con que sabe plantear una unidad didáctica o tema que combine la materia, elementos tecnológicos y un enfoque didáctico adecuado; y menos del $50 \%$ sabe elegir la tecnología que utilizará para complementar lo que enseña, utilizar estrategias didácticas que combinen los contenidos con la tecnología y saber elegir elementos tecnológicos que complementen los contenidos. Llama particularmente la atención el bajo porcentaje de profesores que están "totalmente de acuerdo" y "de acuerdo" en que son capaces de ayudar a sus pares para coordinar el uso de los contenidos, la tecnología y los enfoques didácticos, pues al realizar la sumatoria de las frecuencias de ambas categorías el porcentaje sólo alcanza el 26,6\%.

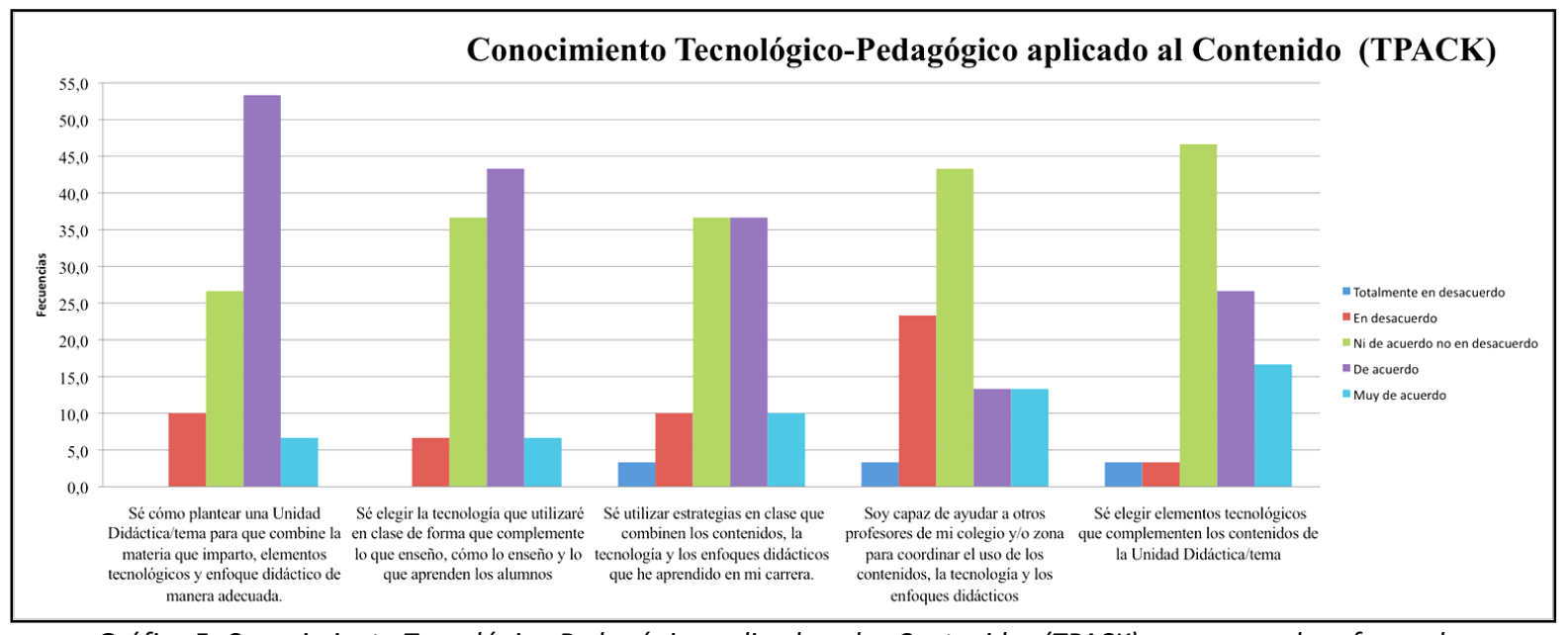

Gráfico 5: Conocimiento Tecnológico-Pedagógico aplicado a los Contenidos (TPACK) que posee el profesorado

\subsection{Significado que le atribuye el profesorado a la integración de las TIC en sus prácticas docentes.}

Con la finalidad de profundizar en el significado que le atribuye el profesorado a la integración de las TIC en sus prácticas docentes, se realizó un focus group con docentes de los niveles educativos de educación infantil y primaria, cuyas preguntas estuvieron vinculadas a las siguientes categorías: Percepción sobre el uso de las TIC - Tecnología y metodología - Aspectos a mejorar. El análisis se llevó a cabo a partir de la contrastación del discurso y los resultados descables se presentan en la tabla 2. 


\begin{tabular}{|c|c|}
\hline Categorías & Resultados \\
\hline $\begin{array}{l}\text { Percepción sobre las } \\
\text { TIC }\end{array}$ & $\begin{array}{l}\text { - La experiencia que han tenido los profesores respecto a la integración de las } \\
\text { TIC en el sus prácticas docentes ha sido positiva y reconocen que estas } \\
\text { herramientas presentan una serie de ventajas, entre ellas la interactividad que } \\
\text { puede tener el alumno con esta herramienta y la motivación que les genera, al } \\
\text { mismo tiempo, son conscientes de que les ayuda a complementar la enseñanza. } \\
\text { - Una de las principales desventajas que presentan las TIC es el tiempo que } \\
\text { tienen que utilizar en la preparación de recursos. Consideran, además, que su } \\
\text { utilización está condicionada al área curricular en la que se quiera } \\
\text { implementar. } \\
\text { - La formación en TIC que han tenido la han hecho por motivaciones e intereses } \\
\text { personales, ya que durante su proceso de formación inicial no tuvieron la } \\
\text { preparación suficiente para desenvolverse eficazmente con estas herramientas. } \\
\text { - Destacan los recursos disponibles en el Colegio y el alto compromiso con que } \\
\text { el claustro ha vivido la incorporación de las TIC, no sólo en lo que respecta a } \\
\text { sus propias clases, sino que también en proyectos de mayor envergadura. }\end{array}$ \\
\hline TIC y Metodología & $\begin{array}{l}\text { - Consideran que han cambiado la metodología de enseñanza y que dicho } \\
\text { cambio se expresa fundamentalmente en las posibilidades que ofrecen estas } \\
\text { herramientas para motivar a los niños, para optimizar los tiempos, para } \\
\text { favorecer el trabajo colaborativo y para que sean los propios estudiantes } \\
\text { quienes busquen la información que se necesita. } \\
\text { - Para ellos el hecho de que los niños logren aprendizajes significativos no está } \\
\text { condicionado por las nuevas tecnologías, sino que depende fundamentalmente } \\
\text { del profesor. }\end{array}$ \\
\hline Aspectos a mejorar & $\begin{array}{l}\text { Se establecen dos niveles en cuanto a los aspectos a mejorar, uno vinculado al } \\
\text { ámbito administrativo y otro a las prácticas docentes: } \\
\text { - A nivel administrativo se presenta la necesidad de un profesional exclusivo } \\
\text { para las cuestiones informáticas, ya que las dificultades que se presentan les } \\
\text { agobian y ha generado molestias. } \\
\text { - A nivel de las prácticas docentes, se alude a la necesidad de apoyarse de la } \\
\text { tecnología para la coordinación del profesorado y a la necesidad de que } \\
\text { algunos docentes cambien su metodología de enseñanza. }\end{array}$ \\
\hline
\end{tabular}

Tabla 2: Significado que le atribuye el profesorado a la incorporación de las TIC en sus prácticas docentes

Se aprecia una experiencia positiva del profesorado respecto a incorporación de las TIC en sus prácticas, reconocen algunas ventajas que estas herramientas les aporta como complemento a sus clases y el interés que genera en los estudiantes, pero consideran que les demanda mucho tiempo preparar recursos y que la incorporación de las mismas está condicionada por el área curricular.

Los profesores perciben un cambio en sus metodologías de enseñanza, pero se centran en estas herramientas como recurso para motivar a los estudiantes y favorecer el trabajo colaborativo. Sin embargo, en el discurso no se evidencia una clara fundamentación sobre los cambios que han generado en sus metodologías de enseñanza.

En cuanto los aspectos a mejorar, se observa que éstos son percibidos desde dos niveles, uno vinculado al ámbito administrativo donde requieren del apoyo permanente de un especialista en informática que les ayude a resolver problemas emergentes con las tecnologías para evitar el agobio, y desde el ámbito de las prácticas docentes los aspectos a mejorar se relacionan con la evaluación y con la necesidad de que agunos profesores cambien su forma de comprender el proceso educativo de acuerdo a los requerimientos actuales. 


\subsection{Percepción del Equipo Directivo sobre la integración de las TIC en el centro educativo.}

Con la finalidad de complementar los resultados obtenidos anteriormente entrevistamos a dos miembros del equipo directivo. Ambas entrevistas fueron contrastadas, y los resultados se presentan agrupados en las categorías semánticas establecidas: Percepción sobre las TICAspectpos a mejorar- Características del centro respecto a las TIC - Políticas de incorporación de las TIC (veáse tabla 3)..

\begin{tabular}{|c|c|}
\hline Categorías & Resultados \\
\hline $\begin{array}{l}\text { Percepción } \\
\text { sobre las TIC }\end{array}$ & $\begin{array}{l}\text { - Desde el punto de vista pedagógico para estos directivos las TIC se constituyen en } \\
\text { instrumentos que permiten plantearse nuevos objetivos educativos y nuevas formas de } \\
\text { abordar la información y el conocimiento, al mismo tiempo pueden ayudar a los } \\
\text { docentes a ser más efectivos y eficaces en el desempeño de sus tareas docentes y, } \\
\text { específicamente en las actividades de aprendizaje. } \\
\text { - La utilización de las nuevas tecnologías y otras acciones emprendidas a nivel curricular } \\
\text { han contribuido al mejoramiento en el rendimiento de los niños y niñas, lo que ha } \\
\text { llevado a un aumento en la demanda social del Centro. }\end{array}$ \\
\hline $\begin{array}{c}\text { Aspectos a } \\
\text { mejorar }\end{array}$ & $\begin{array}{l}\text { - Necesidad de contar con un experto en informática que les ayude a hacerle mantención a } \\
\text { los equipos y resolver problemas que se les van presentando con las tecnologías. } \\
\text { - A futuro se percibe la necesidad de remirar los enfoques de enseñanza, pues, las } \\
\text { tecnologías utilizadas como recurso de motivación pueden transformarse en algo } \\
\text { habitual y perder esa condición que actualmente ofrecen. }\end{array}$ \\
\hline $\begin{array}{l}\text { Características } \\
\text { del centro } \\
\text { respecto a las } \\
\text { TIC }\end{array}$ & $\begin{array}{l}\text { - En el centro educativo existe la figura del Coordinador TIC, con funciones de } \\
\text { administrador del software, formación del profesorado y técnico. } \\
\text { - El centro tiene la dotación de infraestructura recogida en la convocatoria de Centro } \\
\text { Educativo Inteligente. } \\
\text { - El centro es autónomo para gestionar su formación, aunque el formador debe ser externo } \\
\text { - normativa legal- y actualmente la formación del profesorado se focaliza hacia dos } \\
\text { líneas: inglés y TIC. }\end{array}$ \\
\hline $\begin{array}{c}\text { Políticas de } \\
\text { incorporación } \\
\text { de las TIC }\end{array}$ & $\begin{array}{l}\text { - Desde los inicios del Colegio se han estado implementando las TIC en forma } \\
\text { permanente, tanto en los procesos de gestión como en los académicos. No obstante, } \\
\text { dentro del establecimiento no existe un documento que contenga las políticas de } \\
\text { implementación de las TIC o un plan específico en este aspecto, pero dentro del } \\
\text { Proyecto Curricular de Centro se habla en forma explícita de la competencia referida al } \\
\text { Tratamiento de la Información y Competencia Digital. }\end{array}$ \\
\hline
\end{tabular}

Tabla 3. Percepción de los Directivos sobre la integración de las TIC en el Centro.

\subsection{Convergencias entre el significado que le atribuyen el profesorado a las TIC y el significado del equipo directivo.}

En función de los resultados obtenidos respecto de los significados que construye el profesorado y el equipo directivo en torno a la implementación de las TIC en los procesos administrativos y académicos, se puede decir que existen una serie de convergencias entre ambos actores, evidencia que se presenta por cada categoría con apoyo de citas textuales extraídas desde el discurso de cada uno de los actores.

\subsubsection{Percepción sobre las TIC incorporadas al proceso de enseñanza.}


Ambos actores coinciden en que las tecnologías son herramientas muy útiles para apoyar, facilitar la labor docente y ampliar el campo de conocimientos. No obstante, algunos docentes y directivos advierten la necesidad de tener en cuenta que sólo son herramientas que complementan la labor docente. Uno de los recursos tecnológicos que más destacan es la pizarra digital (véase fig. 2).

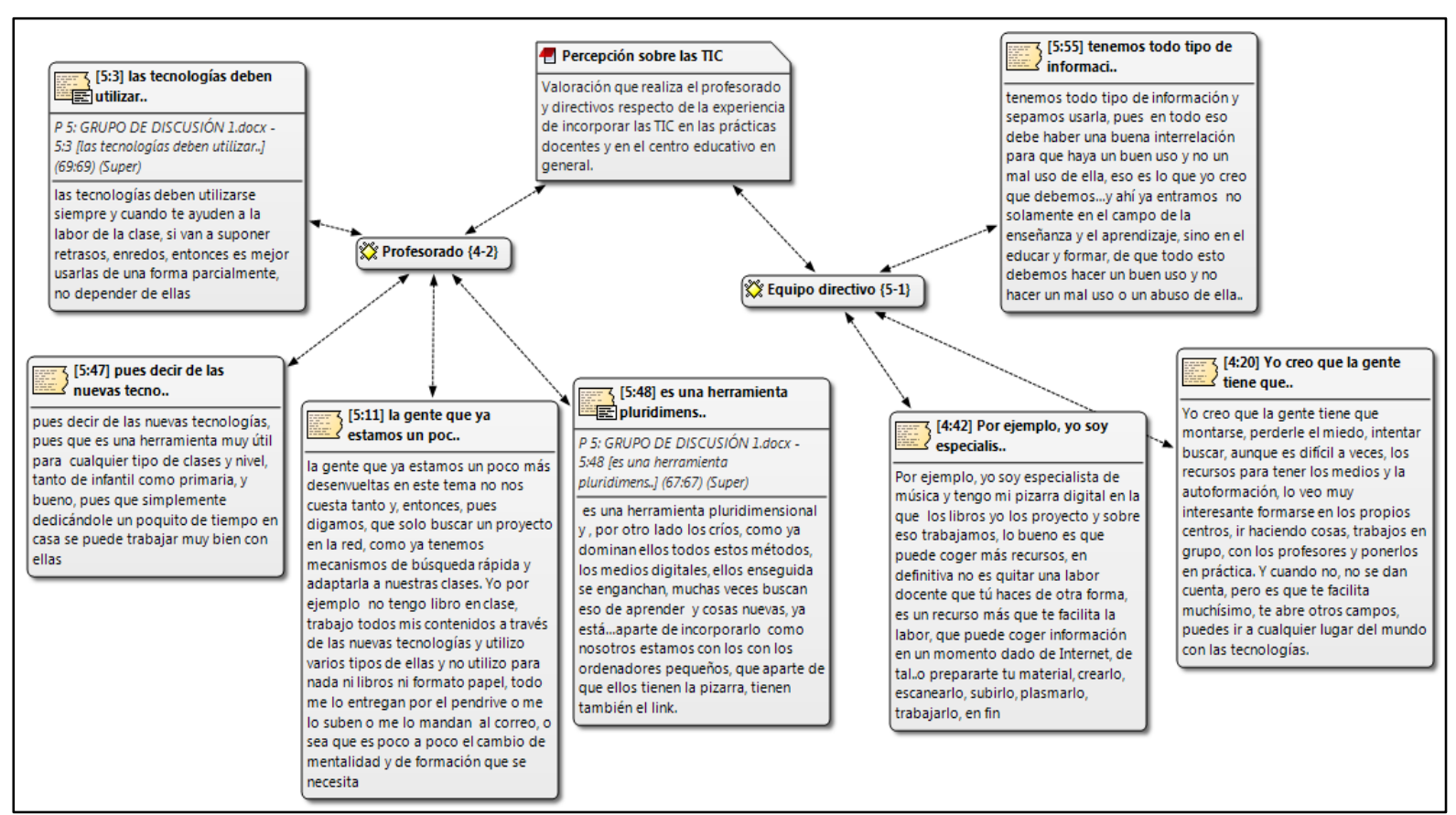

Figura 2: Percepción de los actores sobre las TIC aplicadas al proceso educativo

\subsubsection{Aspectos a mejorar}

A nivel administrativo, ambos actores manifiestan la necesidad de que el centro educativo cuente con una persona experta en informática de manera permanente, para que les ayude a resolver problemas que se les van presentando con la tecnología, pues, tienen un apoyo externo pero les genera cierta complicación. Por otro lado, surge la necesidad de mantener la condición de Centro Inteligente, para lo cual requieren mejorar en forma sistemática la utilización de estas herramientas en el aula.

A nivel de las prácticas pedagógicas se destaca, como aspecto a mejorar, las prácticas docentes de aquellos profesores que continúan realizando sus clases bajo un modelo tradicional, donde sólo incorporan la tecnología pero en forma estática, sin mayor interactividad de los niños y niñas con estas herramientas (veáse fig.3). 


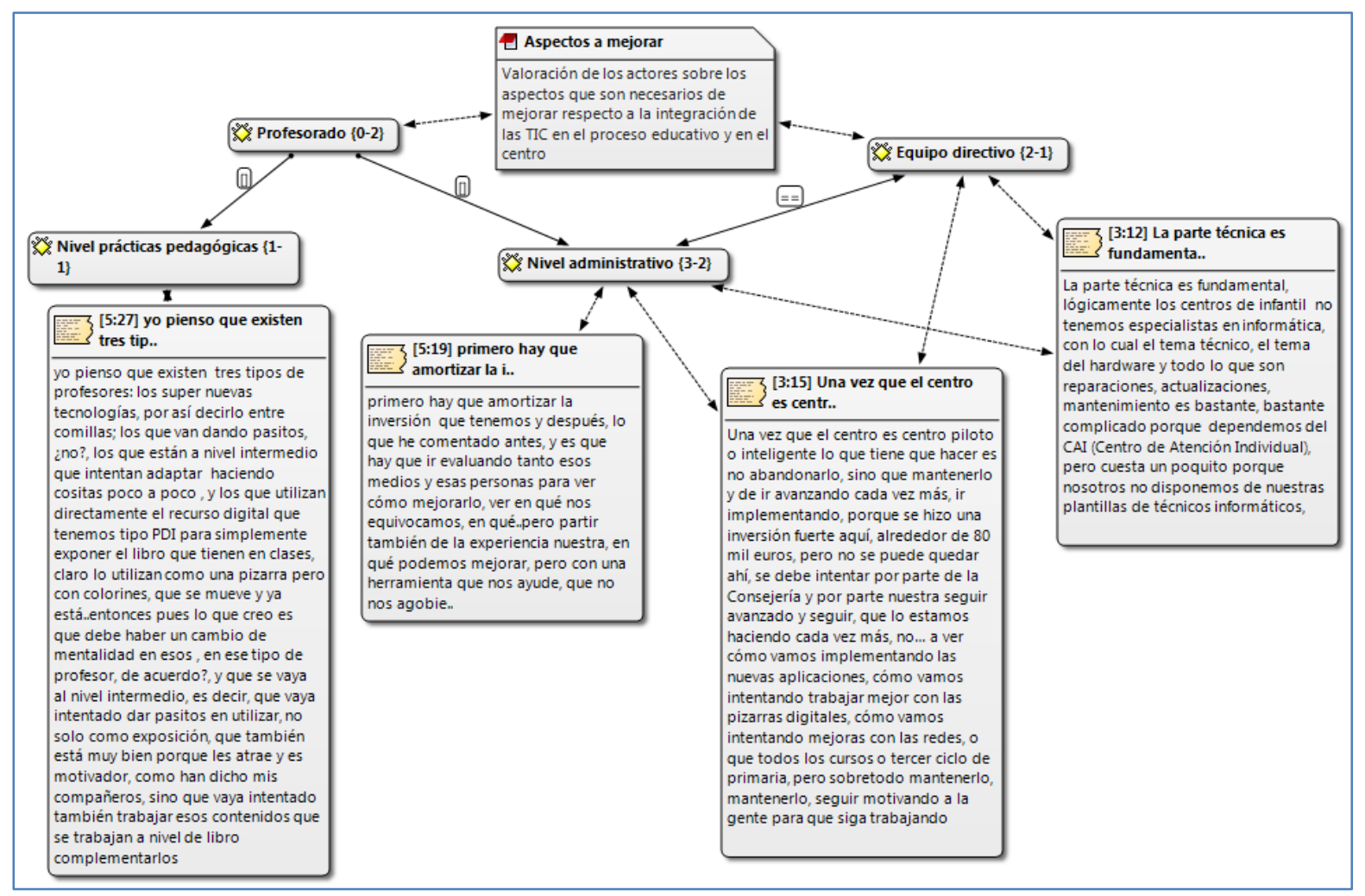

Figura 2.Opinión de los actores sobre los aspectos a mejorar frente a las TIC.

\subsubsection{TIC y Metodología}

Si bien es cierto el profesorado no manifiesta explícitamente haber cambiado sus metodologías de enseñanza a partir del uso de las TIC, si proporcionan ciertas ideas referidas a este aspecto cuando aluden a favorecer la motivación, el aprendizaje colaborativo y a la búsqueda de información por parte de los niños y niñas haciendo uso de las tecnologías, donde nuevamente se menciona la pizarra digital como principal herramienta, comienzando con ella a partir de los 3 años (veáse fig. 4). Mientras que los directivos señalan que ven cambios en los profesores, como se evidencia en la siguente cita:

P 4: ENTREVISTA JEFA DE ESTUDIOS.docx - 4:44 [al principio se agobiaban, por..] (47:47) (Super)

Códigos: [Cambios en la metodología - Familia: Tic y Metodología]

"al principio se agobiaban, por así decirlo, ahora están demandando hacer otras cosas porque se les ha quedado corto, entonces son los que ahora están..es que yo ahora quiero hacer una plataforma Moodle, quiero abrirme un blog..no si la tutoría virtual ya la hago, pero quiero hacérmela yo, gestionármela yo, cerrármela yo, o sea, son los mismos profesores que yo veo y me digo...jmadre mía! Es que hace unos años no nos acordamos que todos teníamos un poquito de miedo..pues ahora cómo van demandando!. 


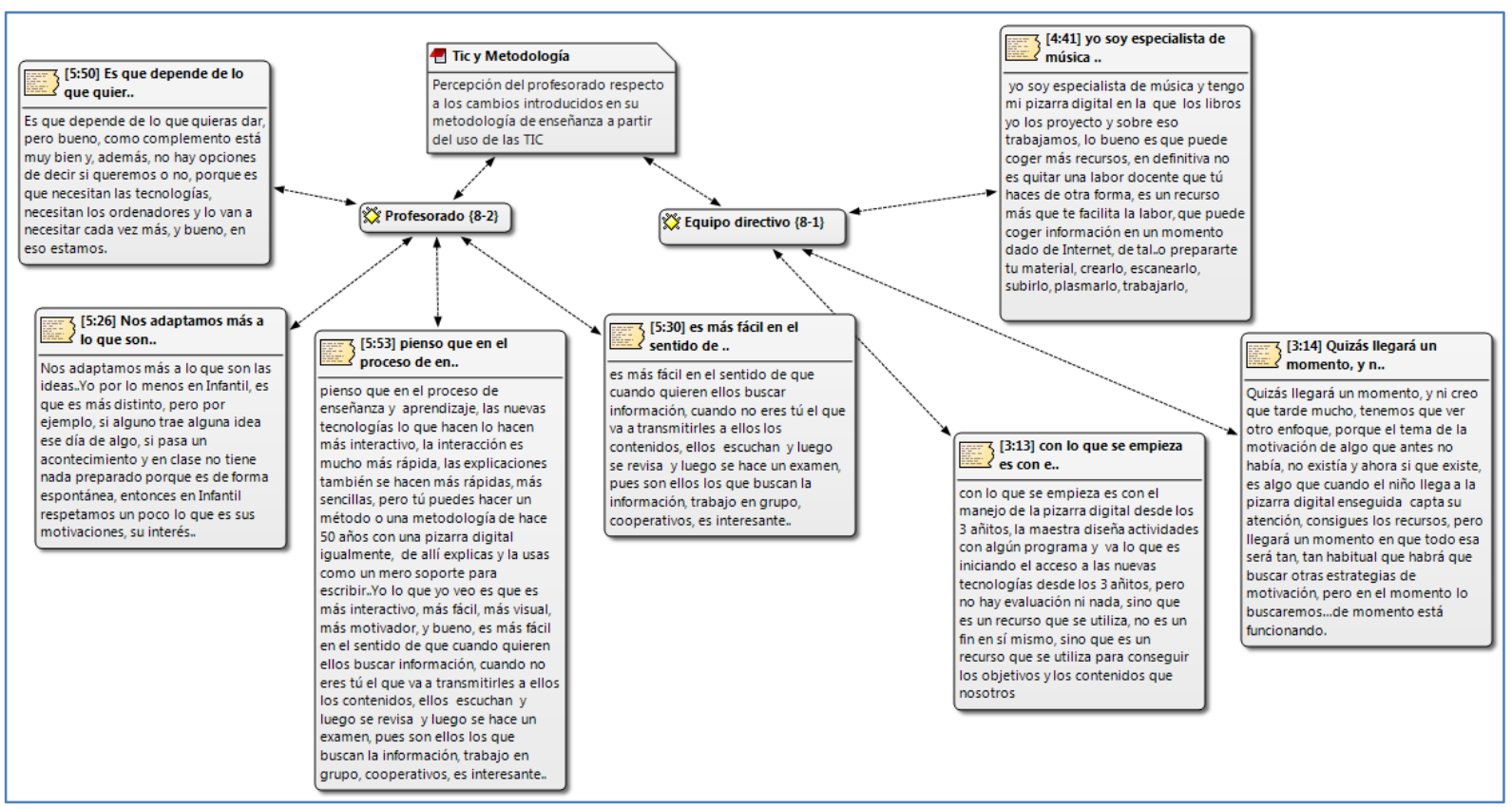

Figura 4: Percepción de los actores sobre las TIC y las metodologías de enseñanza

\subsubsection{Opinión sobre las características del Centro Educativo}

Dentro de las características que subrayan ambos actores es el compromiso del claustro por incorporar estas herramientas en su labor docente, aspecto que generó la motivación por presentarse a la postulación para ser Centro Educativo Inteligente. Por otra parte, tanto el profesorado como los directivos valoran de manera significativa la importante dotación en tecnología con las que cuenta el Colegio (véase fig. 5).

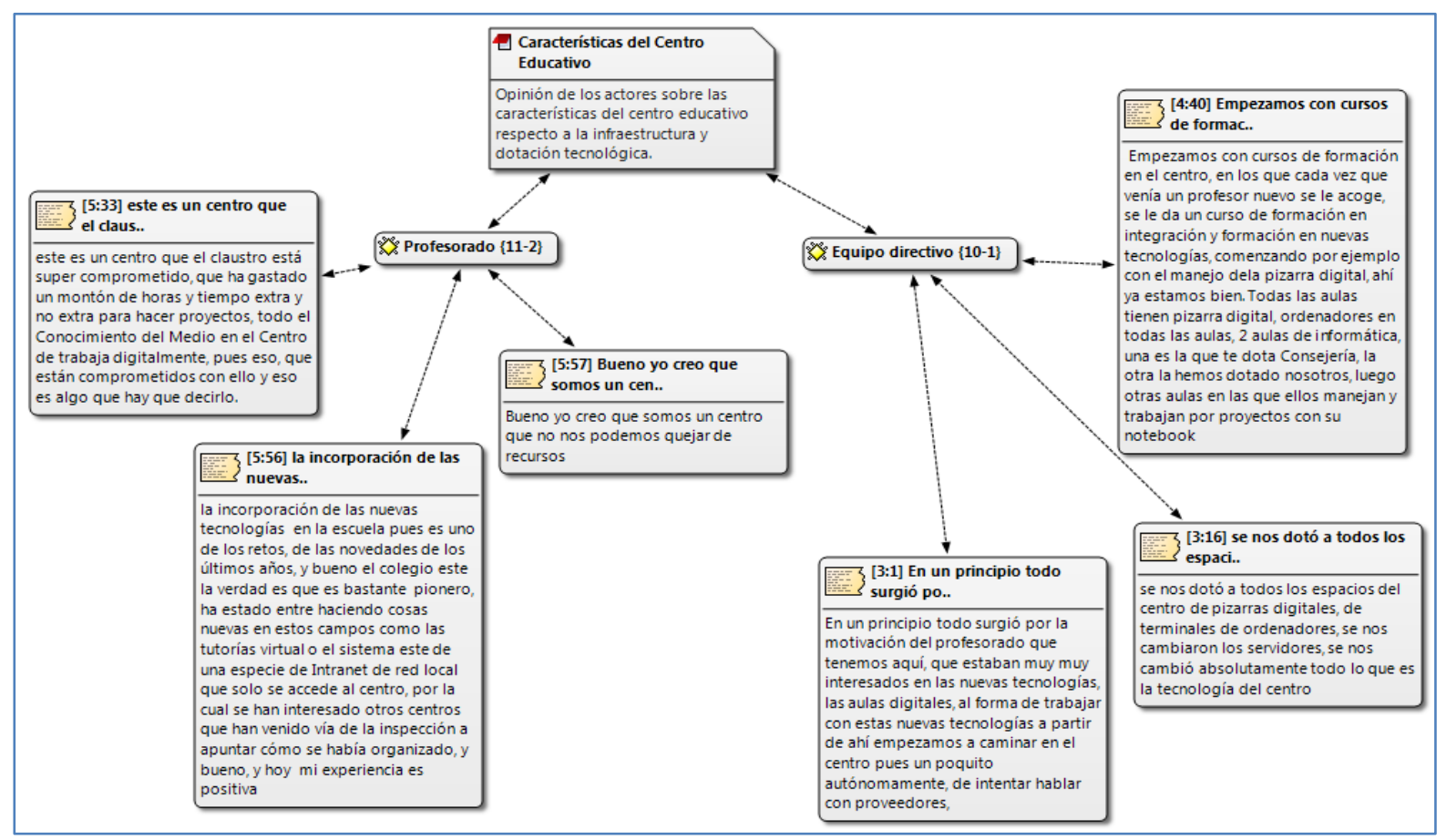

Figura 5: Opinión de los actores frente a las características del Centro Educativo

\section{DISCUSIÓN Y CONCLUSIONES}


La implementación de políticas educativas es necesaria para el desarrollo y delimitación de escenarios de aprendizaje vinculados a los nuevos contextos sociales y culturales (Groff, 2013). En este sentido, la implementación de políticas educativas focalizadas en las TIC en los centros educativos ha demandado nuevos retos y exigencias a todos los actores implicados en el proceso formativo, pero más específicamente al profesorado, pues, su actitud frente a estas herramientas y las competencias que posea para manejarlas y aplicarlas en el proceso educativo son factores que determinarán el éxito de la integración de éstas en el aula (Silva \& Astudillo, 2012). Al respecto, es importante destacar que el desafío para los docentes se presenta principalmente en la capacidad que demuestre para integrar sus conocimientos pedagógicos y tecnológicos en el ámbito disciplinario en función de lograr una buena práctica con TIC.

Uno de los objetivos que nos planteamos fue detectar el conocimiento tecnológico, pedagógico y disciplinario de los docentes en la integración educativa de las TIC en un centro concreto. Al respecto, los resultados generales obtenidos indican que el profesorado autopercibe un mayor dominio de sus conocimientos pedagógicos y disciplinares que tecnológicos, confirmando así resultados de otros estudios realizados en el contexto nacional sobre el dominio de conocimientos tecnológicos por parte de los docentes de primaria (Suárez, Almerch, Díaz-García \& Fernández, 2012; Valverde, Garrido \& Sosa, 2010). Sin embargo, llama la atención este resultado dada la oportunidad que les ofrece el centro educativo para acceder fácilmente a las herramientas tecnológicas, de mostrar una preocupación permanente por capacitarse y de contar con el apoyo de los directivos.

Por otro lado, los resultados permiten sostener que los profesores y directivos en forma progresiva se han ido apropiando de estas herramientas, lo que ha llevado al claustro a asumir un alto grado de compromiso para integrarlas en sus prácticas, demostrando de este modo una actitud positiva frente a las TIC (Domínguez, 2011; Marín \& Reche, 2012; Ramírez, Cañedo \& Clemente, 2012), aunque también advierten ciertas desventajas que presentan estas herramientas, entre ellas el tiempo que requieren para la preparación de recursos y las características del área curricular donde se quieran implementar

Se trata de un centro educativo que, en teoría, tiene un bagaje propio que puede hacernos pensar que se da en su seno un escenario de aprendizaje óptimo en cuanto a los elementos que deben configurar una integración efectiva de las TIC, pero, Ilama la atención, por ejemplo, que sólo la mitad del profesorado (53\%) esté de acuerdo con que sabe plantear una unidad didáctica o tema que combine la materia, elementos tecnológicos y un enfoque didáctico adecuado; que menos de la mitad sepa elegir la tecnología que utilizará para complementar lo que enseña y utilizar estrategias didácticas que combinen los contenidos con la tecnología, a pesar de las instancias de perfeccionamiento y capacitación que han tenido, gestionadas desde el propio centro en función de sus necesidades reales. Cabría preguntarse al respecto sobre la efectividad de dichos procesos formativos.

El liderazgo y apoyo del equipo directivo y el Coordinador TIC se constituyen en elementos clave para la integración de las TIC dentro de las prácticas pedagógicas de los docentes (Valverde, Garrido \& Sosa, 2010), y aunque dentro del Proyecto Educativo del Centro estudiado no existe una política explícita sobre TIC, estas han sido fomentadas desde la dirección y asumidas en la cotidianidad del quehacer administrativo y pedagógico, lo cual es refrendado por el profesorado. 
Finalmente, se destaca que desde la perspectiva del Equipo Directivo, la utilización de las nuevas tecnologías y otras acciones emprendidas a nivel curricular vinculadas a las TIC han contribuido al mejoramiento en el rendimiento de los niños y niñas, lo que ha generado un aumento en la demanda social del Centro. No obstante, conviene subrayar que esta afirmación no posee un sustento teórico y empírico de base, pues, no se evidencia en este contexto educativo mecanismos de evaluación y/o seguimiento sobre el impacto que están teniendo estas herramientas en el aprendizaje de los estudiantes. Por tal razón, consideramos necesario que se lleven a cabo estudios sobre los procesos desarrollados en los CEIP que participan en programas impulsados desde los gobiernos autonómicos, como ha sido el caso de los Centros Educativos Inteligentes, pues, se requiere investigar y transparentar la forma sobre cómo se han ido implementado este tipo de políticas educativas, el impacto que han tenido a nivel de estructura del centro, en los aprendizajes de los estudiantes y en las innovaciones desarrolladas, estudios todos ellos que no se han realizado hasta la fecha.

\section{AGRADECIMIENTOS.}

El presente trabajo se enmarca en el seno del Grupo de Investigación "EDUTIC-ADEI" (Ref.: Vigrob039), del Grupo de Investigación e Innovación en Tecnología Educativa (GITE) "EDUTIC-ADEI-EDAFIS" (Ref. GITE-09004-UA), y del Programa de Redes de investigación en docencia universitaria del ICE, todos ellos de la Universidad de Alicante (UA); del proyecto Instituto Superior de Investigación Cooperativa IVITRA(Ref.: ISIC/2012/022; http://www.ivitra.ua.es) y del Proyecto DIGICOTRACAM ("Programa Prometeo de la Generalitat Valenciana para Grupos de Investigación en I+D de Excelencia", Ref.: Prometeo-2009-042, cofinanciado por el FEDER de la UE) y MICINN FFI2009-13065 y FFI2010-09064.

\section{REFERENCIAS BIBLIOGRÁFICAS}

Bisquerra,R. (2009). Metodología de la Investigación Educativa. Madrid:Editorial La Muralla.

DOGV (2010). RESOLUCIÓN de 24 de septiembre de 2010, de la Dirección General de Innovación Tecnológica Educativa, por la que se determinan los centros autorizados en el programa de pilotaje para el curso 2010-2011, de centros educativos inteligentes. Recuperado de http://www.docv.gva.es/datos/2010/10/05/pdf/2010 10500.pdf

Domínguez, R. (2011). Formación, competencia y actitudes sobre las TIC del profesorado de secundaria: Un instrumento de evaluación. Etic@Net [publicación en línea], IX(10). Recuperado de http://goo.gl/JKQDcO

Gallardo, B., Suarez, J. \& Almerich, G. (2006). La influencia de las actitudes de los profesores en el uso de las nuevas tecnologías. Revista Española de Pedagogía, 233, 45-66. Recuperado de http://goo.gl/k1hTL

Groff, J. (2013). Dynamic Systems Modeling in Educational System Design \& Policy. Journal Of New Approaches In Educational Research, 2(2), 72-81. doi:10.7821/naer.2.2.72-81. Recuperado de http://naerjournal.ua.es/article/view/v2n2-3 
Harris, J., Mishra, P. \& Koehler, M. (2009).Teachers' Technological Pedagogical Content Knowledge and Learning Activity Types: Curriculum-based Technology Integration Reframed. Journal of Research on Technology in Education, 41(4), 393-416. Recuperado de http://goo.gl/fVQS2

López, A. (2011). Usos y actitudes de estudiantes universitarios futuros profesores sobre Tecnologías de la Información y la Comunicación (TIC) y recursos sociales de internet. Questions. Revista Especializada de Periodismo y Comunicación, 1(31). Recuperado de http://goo.gl/NgKxN

Marín, V. \& Reche, E. (2012). Universidad 2.0: actitudes y aptitudes ante las TIC del alumnado de nuevo ingreso de la escuela universitaria de magisterio de la UCO. Pixel-Bit. Revista de Medios de Comunicación, 40, 197-211.

Mischra, P. \& Koehler, M. (2006) Technological Pedagogical Content Knowledge: A Framework for Teacher Knowledge. Teachers College Record, 108 (6),1017-1054. Recuperado de http://goo.gl/V7zf2

Ramírez, E. , Cañedo, I. \& Clemente, M. (2012). Las actitudes y creencias de los profesores de secundaria sobre el uso de Internet en el aula. Comunicar, 38 (XIX),147-155. doi: 10.3916/38-2012-03-06

Sáez Alonso, R. \& Touriñan, J.M. (2012). Teoría de la Educación, metodología y focalizaciones. España: Netbiblo. S.L.

Silva, J. \& Astudillo, A. (2012). Inserción de TIC en la Formación Inicial Docente: Barreras y Oportunidades. Revista Iberoamericana de Educación, 4(58). Recuperado de http://www.rieoei.org/deloslectores/4557Silva.pdf

Sing, Ch., Hwee, J. \& Tsai, Ch. (2013). A Review of Technological Pedagogical Content Knowledge.Educational Technology \& Society, 16(2), 31-51.

Schmidt, D. A., Baran, E., Thompson, A. D., Mishra, P., Koehler, M. J., \& Shin, T. S. (2009). Technological pedagogical content knowledge (TPACK): The development and validation of an assessment instrument for preservice teachers. Journal of Research on Computing in Education, 42(2), 123-149.Recuperado de http://learnonline.canberra.edu.au/pluginfile.php/491591/mod page/content/1/TPACK $\underline{\mathrm{UC} / \mathrm{pdf} / \text { tpack4 preservice2.pdf }}$

Suárez, J., Almerch, G., Díaz-García, M. \& Fernández, R.(2012). Competencias del profesorado en las TIC. Influencia de factores personales y contextuales. Univ. Psychol. [online], 11(1), 293-309.

Tejedor, F.J. \& García-Valcárcel, A. (2006). Competencias de los profesores para el uso de las TIC en la enseñanza. Análisis de sus conocimientos y actitudes. Revista Española de Pedagogía, 233, 21-44. Recuperado de http://goo.gl/2bLaO

Valverde, J., Garrido, Ma.C. \& Fernández, Mạ.R. (2010). Enseñar y aprender con tecnologías: un modelo teórico para las buenas prácticas educativas con TIC, Teoría de la Educación. Educación y Cultura en la Sociedad de la Información, 11(1), 203-229.

Valverde, J., Garrido, Mạ.C. \& Sosa-Díaz, Mạ.J. (2010). Políticas educativas para la integración 
de las TIC en Extremadura y sus efectos sobre la innovación didáctica y el proceso enseñanza-aprendizaje: la percepción del profesorado, Revista de Educación, 352, 99-124.

\section{Para citar este artículo:}

Roig R. \& Carolina Flores, C. Conocimiento tecnológico, pedagógico y disciplinario del profesorado: el caso de un centro educativo inteligente. EDUTEC, Revista Electrónica de Tecnología Educativa, 47. Recuperado el dd/mm/aa de http://edutec.rediris.es/Revelec2/Revelec47/n47 Roig-Flores.html 